\title{
Certain Integral Operator Related to the Hurwitz-Lerch Zeta Function
}

\author{
Xiao-Yuan Wang $\mathbb{D}^{1},{ }^{1}$ Lei Shi $\mathbb{D}{ }^{2}{ }^{2}$ and Zhi-Ren Wang ${ }^{1}$ \\ ${ }^{1}$ College of Science, Yanshan University, Hebei, Qinhuangdao 066004, China \\ ${ }^{2}$ School of Mathematics and Statistics, Anyang Normal University, Henan, Anyang 455000, China
}

Correspondence should be addressed to Xiao-Yuan Wang; mewangxiaoyuan@163.com

Received 16 August 2017; Accepted 6 March 2018; Published 8 April 2018

Academic Editor: Pranay Goswami

Copyright (C) 2018 Xiao-Yuan Wang et al. This is an open access article distributed under the Creative Commons Attribution License, which permits unrestricted use, distribution, and reproduction in any medium, provided the original work is properly cited.

The aim of the present paper is to investigate several third-order differential subordinations, differential superordination properties, and sandwich-type theorems of an integral operator $\mathscr{W}_{s, b} f(z)$ involving the Hurwitz-Lerch Zeta function. We make some applications of the operator $\mathscr{W}_{s, b} f(z)$ for meromorphic functions.

\section{Introduction}

Denote by $\mathscr{H}(\mathbb{U})$ the class of functions analytic in the unite disk

$$
\mathbb{U}=\{z: z \in \mathbb{C},|z|<1\}
$$

of the form

$$
\begin{array}{r}
\mathscr{H}[a, n]=\left\{f: f \in \mathscr{H}(\mathbb{U}), \quad f(z)=a+\sum_{k=n}^{\infty} a_{k} z^{k}\right\} \\
(a \in \mathbb{C} ; n \in \mathbb{N}=\{1,2, \ldots\})
\end{array}
$$

and let $\mathscr{H}=\mathscr{H}[1,1]$

For two functions $f(z)$ and $g(z)$ to be analytic in $\mathbb{U}, f(z)$ is said to be subordinate to $g(z)$ in $\mathbb{U}$ and written by

$$
f(z) \prec g(z) \quad(z \in \mathbb{U}),
$$

if there exists a Schwarz function $\omega(z)$, which is analytic in $\mathbb{U}$, with

$$
\begin{aligned}
\omega(0) & =0, \\
|\omega(z)| & <1,
\end{aligned}
$$

such that

$$
f(z)=g(\omega(z)) \quad(z \in \mathbb{U}) .
$$

It is generally known that

$$
\begin{aligned}
& f(z) \prec g(z) \quad(z \in \mathbb{U}) \Longrightarrow \\
& f(0)=g(0), \quad f(\mathbb{U}) \subset g(\mathbb{U}) .
\end{aligned}
$$

Furthermore, if the function $g(z)$ is univalent in $\mathbb{U}$, then

$$
\begin{aligned}
& f(z) \prec g(z) \quad(z \in \mathbb{U}) \Leftrightarrow \\
& f(0)=g(0), \quad f(\mathbb{U}) \subset g(\mathbb{U}) .
\end{aligned}
$$

Denote by $Q$ the set of functions $q(z)$ that are analytic and univalent on $\overline{\mathbb{U}} \backslash \mathscr{E}(q)$, where

$$
\mathscr{E}(q)=\left\{\zeta \in \partial \mathbb{U}: \lim _{z \rightarrow \zeta} q(z)=\infty\right\}
$$

are such that $\min \left|q^{\prime}(\zeta)\right|=\varepsilon>0$ for $\zeta \in \partial \mathbb{U} \backslash \mathscr{E}(q)$. Furthermore, let

$$
\begin{aligned}
Q(a) & =\{q(z) \in Q: q(0)=a\}, \\
Q_{1} & =Q(1) .
\end{aligned}
$$

Denote by $\mathscr{A}^{*}$ the class of functions of the form

$$
f(z)=\frac{1}{z}+\sum_{n=1}^{\infty} a_{n} z^{n},
$$


which are analytic in the punctured unit disk

$$
\mathbb{U}^{*}=\{z \in \mathbb{C}, 0<|z|<1\}=\mathbb{U} \backslash\{0\} .
$$

We recall the general Hurwitz-Lerch Zeta function $\Phi(z, s, a)$ (see, e.g., [1, p. 121] and [2, p. 194]) defined by

$$
\begin{aligned}
& \Phi(z, s, a):=\sum_{k=0}^{\infty} \frac{z^{k}}{(k+a)^{s}} \\
& \left(a \in \mathbb{C} \backslash \mathbb{Z}_{0}^{-} ; s \in \mathbb{C} \text { when }|z|<1 ; \mathfrak{R}(s)>1 \text { when }|z|=1\right),
\end{aligned}
$$

where

$$
\mathbb{Z}_{0}^{-}:=\mathbb{Z}^{-} \cup\{0\}=\{0,-1,-2, \ldots\} .
$$

In recent years, the general Hurwitz-Lerch Zeta function $\Phi(z, s, a)$ was investigated by many researchers. A huge amount of interesting properties and consequences can be found in, for example, Choi and Srivastava [3], Garg et al. [4], Lin and Srivastava [5], and Srivastava et al. [6].

In 2007, by involving the general Hurwitz-Lerch Zeta function $\Phi(z, s, a)$, Srivastava and Attiya [7] (also see [8-11]) introduced the integral operator

$$
\begin{aligned}
& \mathscr{J}_{s, b} f(z)=z+\sum_{k=2}^{\infty}\left(\frac{1+b}{k+b}\right)^{s} c_{k} z^{k} \\
& \quad\left(b \in \mathbb{C} \backslash \mathbb{Z}^{-} ; s \in \mathbb{C} ; z \in \mathbb{U}\right) .
\end{aligned}
$$

Analogous to abovementioned operator $\mathscr{J}_{s, b} f$, Wang and Shi [12] introduced a new integral operator

$$
\mathscr{W}_{s, b}: \Sigma \longrightarrow \Sigma
$$

defined by

$$
\begin{aligned}
\mathscr{W}_{s, b} f(z) & :=\Theta_{s, b}(z) * f(z) \\
(b & \left.\in \mathbb{C} \backslash\left\{\mathbb{Z}_{0}^{-} \cup\{1\}\right\} ; s \in \mathbb{C} ; f \in \Sigma ; z \in \mathbb{U}^{*}\right),
\end{aligned}
$$

where

$$
\begin{array}{r}
\Theta_{s, b}(z):=(b-1)^{s}\left[\Phi(z, s, b)-b^{-s}+\frac{1}{z(b-1)^{s}}\right] \\
\left(z \in \mathbb{U}^{*}\right),
\end{array}
$$

and “*” denotes the Hadamard product.

From (10), (12), (16), and (17), we easily find that

$$
\mathscr{W}_{s, b} f(z)=\frac{1}{z}+\sum_{k=1}^{\infty}\left(\frac{b-1}{b+k}\right)^{s} a_{k} z^{k}
$$

It is true that $b \in \mathbb{C} \backslash\left\{\mathbb{Z}^{-} \cup\{1\}\right\}$, the integral operator $\mathscr{W}_{s, b}$ defined as

$$
\mathscr{W}_{s, 0} f(z):=\lim _{b \rightarrow 0}\left\{\mathscr{W}_{s, b} f(z)\right\}
$$

We can deduce that

$$
\begin{aligned}
& \mathscr{W}_{0, b} f(z)=f(z), \\
& \mathscr{W}_{-1,0} f(z)=-z f^{\prime}(z), \\
& \mathscr{W}_{-1,-1} f(z)=\frac{f(z)-z f^{\prime}(z)}{2}, \\
& \mathscr{W}_{s, 2} f(z)=\frac{1}{z}+\sum_{k=1}^{\infty}\left(\frac{1}{k+2}\right)^{s} a_{k} z^{k}, \\
& \mathscr{W}_{1, b+1} f(z)=\frac{1}{z}+\sum_{k=1}^{\infty}\left(\frac{b}{k+b+1}\right) a_{k} z^{k} \\
&=\frac{b}{z^{b+1}} \int_{0}^{z} t^{b} f(t) d t \quad(b>0), \\
& \mathscr{W}_{\alpha, \beta+1} f(z)=\frac{\beta^{\alpha}}{\Gamma(s) z^{\beta+1}} \int_{0}^{z} t^{b}\left(\log \frac{z}{t}\right)^{s-1} f(t) d t \\
& \quad(\alpha>0 ; \beta>0) .
\end{aligned}
$$

We also see that

$$
\mathscr{W}_{1, \gamma} f(z)=\frac{\gamma-1}{z^{\gamma}} \int_{0}^{z} t^{\gamma-1} f(t) d t \quad(\Re(\gamma)>1) .
$$

Furthermore, by (18), we observe that

$$
\mathscr{W}_{s+1, b} f(z)=\frac{b-1}{z^{b}} \int_{0}^{z} t^{b-1} \mathscr{W}_{s, b} f(z) d t
$$

$$
(\Re(b)>1) .
$$

Operator (23) was introduced and studied by Alhindi and Darus [13]; operators (24) and (25) were introduced by Lashin [14].

The main purpose of this paper is to derive some thirdorder differential subordination, differential superordination properties, and sandwich-type theorems of the integral operator $\mathscr{W}_{s, b} f(z)$.

\section{Preliminary Results}

We will investigate our main results by using following definitions and lemmas.

Definition 1 (see [15, p. 440, Definition 1]). Suppose that $\Psi: \mathbb{C}^{4} \times \mathbb{U} \rightarrow \mathbb{C}, q(z)$, and $h(z)$ are univalent in $\mathbb{U}$. If $p(z)$ is analytic in $\mathbb{U}$ and satisfies the third-order differential subordination

$$
\psi\left(p(z), z p^{\prime}(z), z^{2} p^{\prime \prime}(z), z^{3} p^{\prime \prime \prime}(z) ; z\right) \prec h(z),
$$

then $p(z)$ is called a solution of the differential subordination. $q(z)$ is called a dominant of the solutions of the differential subordination or more simply a dominant if $p(z) \prec q(z)$ for all $p(z)$ satisfying (28). A dominant $\tilde{q}(z)$ that satisfies

$$
\widetilde{q}(z) \prec q(z),
$$

for all dominants of (28), is called the best dominant of (28). 
As the second-order differential superordinations were introduced and investigated by Miller and Mocanu [16], Tang et al. [17] introduced the following third-order differential superordinations.

Definition 2 (see [17, p. 3, Definition 5]). Suppose that $\psi$ : $\mathbb{C}^{4} \times \mathbb{U} \rightarrow \mathbb{C}$ and the function $h(z)$ is analytic in $\mathbb{U}$. If the functions $p(z)$ and

$$
\psi\left(p(z), z p^{\prime}(z), z^{2} p^{\prime \prime}(z), z^{3} p^{\prime \prime \prime}(z) ; z\right)
$$

are univalent in $\mathbb{U}$ and satisfy the third-order differential superordination

$$
h(z) \prec \psi\left(p(z), z p^{\prime}(z), z^{2} p^{\prime \prime}(z), z^{3} p^{\prime \prime \prime}(z) ; z\right),
$$

then $p(z)$ is called a solution of the differential superordination. An analytic function $q(z)$ is called a subordinant of the solutions of the differential superordination or more simply a subordinant if $q(z) \prec p(z)$ satisfies (31) for $p(z)$ satisfying (31). A univalent subordinant $\tilde{q}(z)$ that satisfies

$$
q(z) \prec \widetilde{q}(z)
$$

for all superordinants $q(z)$ of (31) is said to be the best superordinant.

Lemma 3 (see [18, p. 132], [19, p. 190]). Suppose that $q$ is univalent in the open unit disk $\mathbb{U}$ and $\theta$ and $\phi$ are analytic in a domain $\mathbb{D}$ containing $q(\mathbb{U})$ with $\phi(\omega) \neq 0$ when $\omega \in q(\mathbb{U})$. $\operatorname{Set} \Phi(z)=z q^{\prime}(z) \phi(q(z))$ and $h(z)=\theta(q(z))+\Phi(z)$. Suppose that

(1) $\Phi$ is star-like in $\mathbb{U}$;

(2) $\mathfrak{R}\left(z h^{\prime}(z) / \Phi(z)\right)>0$.

If $p \in \mathscr{H}[q(0), n]$ for some $n \in \mathbb{N}$ with $p(\mathbb{U}) \subset \mathbb{D}$ and

$$
\begin{aligned}
& \theta(p(z))+z p^{\prime}(z) \phi(p(z)) \\
& \quad \prec \theta(q(z))+z q^{\prime}(z) \phi(q(z)),
\end{aligned}
$$

then $p \prec q$ and $q$ is the best dominant.

Lemma 4 (see [20, p. 332]). Suppose that $q$ is univalent in the open unit disk $\mathbb{U}$ and $\theta$ and $\phi$ are analytic in a domain $\mathbb{D}$ containing $q(\mathbb{U})$. Set $\Phi(z)=z q^{\prime}(z) \phi(q(z))$. Suppose that

(1) $\Phi$ is star-like in $\mathbb{U}$;

(2) $\mathfrak{R}\left(\theta^{\prime}(q(z)) / \phi(q(z))\right)>0$.

If $p \in \mathscr{H}[q(0), 1] \cap Q$, with $p(\mathbb{U}) \subseteq \mathbb{D}, \theta(p(z))+z p^{\prime}(z) \phi(p(z))$ is univalent in $\mathbb{U}$, and

$$
\begin{aligned}
& \theta(q(z))+z q^{\prime}(z) \phi(q(z)) \\
& \quad \prec \theta(p(z))+z p^{\prime}(z) \phi(p(z)),
\end{aligned}
$$

then $q \prec p$ and $q$ is the best dominant.

Lemma 5 (see [16, p. 822]). Suppose that $q$ is univalent complex in the open unit disk $\mathbb{U}$ and $\gamma \in \mathbb{C}$, with $\mathfrak{R}(\gamma)>0$. If $p \in \mathscr{H}[q(0), 1] \cap Q, p(z)+\gamma z p^{\prime}(z)$ is univalent in $\mathbb{U}$, and

$$
q(z)+\gamma z q^{\prime}(z) \prec p(z)+\gamma z p^{\prime}(z) \quad(z \in \mathbb{U}),
$$

then $q \prec p$ and $q$ is the best dominant.

\section{Main Results}

In this section, we state several third-order differential subordination and differential superordination results associated with the operator $\mathscr{W}_{s, b} f(z)$.

Theorem 6. Suppose that the function $q \in \mathscr{A}^{*}$ is nonzero univalent in $\mathbb{U}$ with $q(0)=1$ and

$$
\mathfrak{R}\left(1+\frac{z q^{\prime \prime}(z)}{q^{\prime}(z)}-\frac{z q^{\prime}(z)}{q(z)}\right)>0 \quad(z \in \mathbb{U}) .
$$

Let $0 \leq \rho \leq 1$ and $\eta \in \mathbb{C}$. If $f \in \mathscr{H}[0, p]$ satisfies

$$
\begin{aligned}
& {\left[(1-\rho) z \mathscr{W}_{s, b} f(z)+\rho z \mathscr{W}_{s+1, b} f(z)\right] \neq 0 \quad(z \in \mathbb{U})} \\
& \eta\left[\frac{(1-\rho) z\left(\mathscr{W}_{s, b} f(z)\right)^{\prime}+\rho z\left(\mathscr{W}_{s+1, b} f(z)\right)^{\prime}}{(1-\rho) \mathscr{W}_{s, b} f(z)+\rho \mathscr{W}_{s+1, b} f(z)}-1\right] \\
& \quad \prec \frac{z q^{\prime}(z)}{q(z)}
\end{aligned}
$$

then

$$
\left[(1-\rho) z \mathscr{W}_{s, b} f(z)+\rho z \mathscr{W}_{s+1, b} f(z)\right]^{\eta} \prec q(z)
$$

and $q$ is the best dominant in (39). When $\eta=0$ the left hand side expressions in (39) are interpreted as 1.

Proof. Suppose that

$$
p(z):=\left[(1-\rho) z \mathscr{W}_{s, b} f(z)+\rho z \mathscr{W}_{s+1, b} f(z)\right]^{\eta} .
$$

Then $p$ is analytic in $\mathbb{U}$. Logarithmically differentiating both sides of (40) with respect to $z$, we have

$$
\begin{aligned}
& \frac{z p^{\prime}(z)}{p(z)} \\
& \quad \eta\left[\frac{(1-\rho) z\left(\mathscr{W}_{s, b} f(z)\right)^{\prime}+\rho z\left(\mathscr{W}_{s+1, b} f(z)\right)^{\prime}}{(1-\rho) \mathscr{W}_{s, b} f(z)+\rho \mathscr{W}_{s+1, b} f(z)}\right. \\
& -1] .
\end{aligned}
$$

To apply Lemma 3, we set

$$
\begin{aligned}
\theta(\omega) & :=1, \\
\phi(\omega) & :=\frac{1}{\omega}
\end{aligned}
$$

$(\omega \in \mathbb{C} \backslash\{0\})$,

$\Phi(z)=z q^{\prime}(z) \phi(q(z))=\frac{z q^{\prime}(z)}{q(z)} \quad(z \in \mathbb{U})$,

$h(z)=\theta(q(z))+\Phi(z)=1+\frac{z q^{\prime}(z)}{q(z)}$. 
By means of (36) we see that $\Phi(z)$ is univalent star-like in $\mathbb{U}$. Since $h(z)=1+\Phi(z)$, we furthermore get that

$$
\Re\left(\frac{z h^{\prime}(z)}{\Phi(z)}\right)>0 .
$$

By a routine calculation using (40) and (41) we find that

$$
\begin{aligned}
& \theta(p(z))+z p^{\prime}(z) \phi(p(z))=1 \\
& +\eta\left[\frac{(1-\rho) z\left(\mathscr{W}_{s, b} f(z)\right)^{\prime}+\rho z\left(\mathscr{W}_{s+1, b} f(z)\right)^{\prime}}{(1-\rho) \mathscr{W}_{s, b} f(z)+\rho \mathscr{W}_{s+1, b} f(z)}\right. \\
& +1] .
\end{aligned}
$$

Therefore, hypothesis (38) is equivalently written as

$$
\begin{aligned}
& \theta(p(z))+z p^{\prime}(z) \phi(p(z)) \prec 1+\frac{z q^{\prime}(z)}{q(z)} \\
& =\theta(q(z))+z q^{\prime}(z) \phi(q(z)) .
\end{aligned}
$$

We know that condition (33) is also satisfied. From an application of Lemma 3, we have

$$
p(z) \prec q(z) .
$$

Thus, we get the assertions in (39). Thus, the proof of Theorem 6 is completed.

Theorem 7. Suppose that the function $q \in \mathscr{A}^{*}$ is a univalent mapping of $\mathbb{U}$ into the right half plane with $q(0)=1$ and

$$
\Re\left(1+\frac{z q^{\prime \prime}(z)}{q^{\prime}(z)}-\frac{z q^{\prime}(z)}{q(z)}\right)>0 \quad(z \in \mathbb{U}) .
$$

Let $0 \leq \rho \leq 1$ and $\eta \in \mathbb{C}, f \in \mathscr{H}[0, p]$ satisfy

$$
\left[(1-\rho) z \mathscr{W}_{s, b} f(z)+\rho z \mathscr{W}_{s+1, b} f(z)\right] \neq 0 \quad(z \in \mathbb{U}) .
$$

If

$$
\Delta(z) \prec q(z)+\frac{z q^{\prime}(z)}{q(z)},
$$

where

$$
\begin{aligned}
& \Delta(z)=\left[(1-\rho) z \mathscr{W}_{s, b} f(z)+\rho z \mathscr{W}_{s+1, b} f(z)\right]^{\eta} \\
& +\eta\left[\frac{(1-\rho) z\left(\mathscr{W}_{s, b} f(z)\right)^{\prime}+\rho z\left(\mathscr{W}_{s+1, b} f(z)\right)^{\prime}}{(1-\rho) \mathscr{W}_{s, b} f(z)+\rho \mathscr{W}_{s+1, b} f(z)}\right. \\
& -1],
\end{aligned}
$$

then

$$
\left[(1-\rho) z \mathscr{W}_{s, b} f(z)+\rho z \mathscr{W}_{s+1, b} f(z)\right]^{\eta} \prec q(z)
$$

and $q$ is the best dominant in (51). When $\eta=0$, the left hand side expression of (51) is interpreted as 1 .
Proof. Suppose that the function $p(z)$ is defined by (40). If set

$$
\begin{aligned}
& \theta(\omega):=\omega, \\
& \phi(\omega):=\frac{1}{\omega}
\end{aligned}
$$

$(\omega \in \mathbb{C} \backslash\{0\})$,

$$
\begin{aligned}
& \Phi(z)=z q^{\prime}(z) \phi(q(z))=\frac{z q^{\prime}(z)}{q(z)} \quad(z \in \mathbb{U}), \\
& h(z)=\theta(q(z))+\Phi(z)=q(z)+\Phi(z)
\end{aligned}
$$

we easily get

$$
\begin{aligned}
\Re\left(\frac{z h^{\prime}(z)}{\Phi(z)}\right) & =\Re\left(q(z)+1+\frac{z q^{\prime \prime}(z)}{q^{\prime}(z)}-\frac{z q^{\prime}(z)}{q(z)}\right) \\
& >0 \quad(z \in \mathbb{U}) .
\end{aligned}
$$

By virtue of (41), hypothesis (49) can be rewritten as

$$
\begin{aligned}
& \theta(p(z))+z p^{\prime}(z) \phi(p(z)) \\
& \quad \prec \theta(q(z))+z q^{\prime}(z) \phi(q(z)) .
\end{aligned}
$$

Therefore, by making use of Lemma 3, we derive that

$$
p(z) \prec q(z) \quad(z \in \mathbb{U}) .
$$

Thus, the assertion in (49) follows. The proof of Theorem 7 is completed.

Theorem 8. Suppose that the function $q \in \mathscr{A}^{*}$ is a univalent mapping of $\mathbb{U}$ into the right half plane with $q(0)=1$ and satisfies condition

$$
\mathfrak{R}\left(1+\frac{z q^{\prime \prime}(z)}{q^{\prime}(z)}-\frac{z q^{\prime}(z)}{q(z)}\right)>0 \quad(z \in \mathbb{U}) .
$$

Let $0 \leq \rho \leq 1, \eta \in \mathbb{C}$, and $f \in \mathscr{H}[0, p]$ satisfy

$$
\begin{aligned}
& {\left[(1-\rho) z \mathscr{W}_{s, b} f(z)+\rho z \mathscr{W}_{s+1, b} f(z)\right]^{\eta}} \\
& \quad \in \mathscr{H}[1,1] \cap Q .
\end{aligned}
$$

Let function $\Delta(z)$ be univalent in $\mathbb{U}$, where $\Delta(z)$ is defined by (50). If

$$
q(z)+\frac{z q^{\prime}(z)}{q(z)} \prec \Delta(z),
$$

then

$$
q(z) \prec\left[(1-\rho) z \mathscr{W}_{s, b} f(z)+\rho z \mathscr{W}_{s+1, b} f(z)\right]^{\eta}
$$

and $q$ is the best subordinant in (59). When $\eta=0$, the left hand side expressions of (59) are interpreted as 1. 
Proof. By putting

$$
\begin{aligned}
& \theta(\omega):=\omega, \\
& \phi(\omega):=\frac{1}{\omega}
\end{aligned}
$$$$
(\omega \in \mathbb{C} \backslash\{0\}),
$$

$$
\Phi(z)=z q^{\prime}(z) \phi(q(z))=\frac{z q^{\prime}(z)}{q(z)} \quad(z \in \mathbb{U}),
$$

obviously, $\Phi$ is star-like in $\mathbb{U}$ and

$$
\mathfrak{R}\left(\frac{\theta^{\prime}(q(z))}{\phi(q(z))}\right)=\mathfrak{R}(q(z)) \quad(z \in \mathbb{U}) .
$$

Suppose that function $p$ is defined by (40). By simple calculation, from (41), we know that

$$
\theta(p(z))+z p^{\prime}(z) \phi(p(z))=\Delta(z) .
$$

Hence, condition (58) can be equivalently written as

$$
\begin{aligned}
& \theta(q(z))+z q^{\prime}(z) \phi(q(z)) \\
& \quad \prec \theta(p(z))+z p^{\prime}(z) \phi(p(z)) .
\end{aligned}
$$

Therefore, by Lemma 4, we have

$$
q(z) \prec p(z) \quad(z \in \mathbb{U})
$$

and $q$ is the best subordinant. The proof of Theorem 8 is completed.

Theorem 9. Suppose that $0 \leq \rho \leq 1, \alpha, \eta \in \mathbb{C}$, the function $q \in \mathscr{A}^{*}$ is univalent in $\mathbb{U}$, and

$$
\Re\left(1+\frac{z q^{\prime \prime}(z)}{q^{\prime}(z)}\right)>\max \{0,-\Re(\alpha)\} .
$$

Let $f \in \mathscr{H}[0, p]$ satisfy

$$
\left[(1-\rho) z \mathscr{W}_{s, b} f(z)+\rho z \mathscr{W}_{s+1, b} f(z)\right] \neq 0 \quad(z \in \mathbb{U}) .
$$

Denote by

$$
\begin{aligned}
& \Xi(z)=\left[(1-\rho) z \mathscr{W}_{s, b} f(z)+\rho z \mathscr{W}_{s+1, b} f(z)\right]^{\eta} \times\{\alpha \\
& +\eta\left[\frac{(1-\rho) z\left(\mathscr{W}_{s, b} f(z)\right)^{\prime}+\rho z\left(\mathscr{W}_{s+1, b} f(z)\right)^{\prime}}{(1-\rho) \mathscr{W}_{s, b} f(z)+\rho \mathscr{W}_{s+1, b} f(z)}\right. \\
& -1]\} \quad(z \in \mathbb{U}) .
\end{aligned}
$$

If

$$
\Xi(z) \prec \alpha q(z)+z q^{\prime}(z)
$$

then

$$
\left[(1-\rho) z \mathscr{W}_{s, b} f(z)+\rho z \mathscr{W}_{s+1, b} f(z)\right]^{\eta} \leq q(z)
$$

and $q$ is the best dominant in (69). When $\eta=0$, the left side hand expressions of (69) are interpreted as 1.
Proof. Suppose that function $p(z)$ is defined by (40). Making using of (41), we have

$$
z p^{\prime}(z)=\eta p(z)
$$$$
\cdot\left[\frac{(1-\rho) z\left(\mathscr{W}_{s, b} f(z)\right)^{\prime}+\rho z\left(\mathscr{W}_{s+1, b} f(z)\right)^{\prime}}{(1-\rho) \mathscr{W}_{s, b} f(z)+\rho \mathscr{W}_{s+1, b} f(z)}\right.
$$<smiles>IC1CCCCC1</smiles>

Therefore, by putting

$$
\begin{aligned}
& \theta(\omega):=\alpha \omega, \\
& \phi(\omega):=1
\end{aligned}
$$

$(\omega \in \mathbb{C})$

$$
\begin{aligned}
& \Phi(z)=z q^{\prime}(z) \phi(q(z))=z q^{\prime}(z) \quad(z \in \mathbb{U}), \\
& h(z)=\theta(q(z))+\Phi(z)=\alpha q(z)+z q^{\prime}(z),
\end{aligned}
$$

obviously, $\Phi$ is star-like in $\mathbb{U}$ and

$$
\mathfrak{R}\left(\frac{z h^{\prime}(z)}{\Phi(z)}\right)=\mathfrak{R}\left(\alpha+1+\frac{z q^{\prime \prime}(z)}{q^{\prime}(z)}\right)>0 .
$$

Furthermore, by substituting the expression for $p(z), z p^{\prime}(z)$ from (40) and (70), respectively, we get

$$
\begin{aligned}
& \theta(p(z))+z p^{\prime}(z) \phi(p(z)) \\
& \quad=\alpha(p(z))+z p^{\prime}(z) \phi(p(z))=\Xi(z),
\end{aligned}
$$

where $\Xi(z)$ is given by (67). Hypothesis (68) can be equivalently written as

$$
\begin{aligned}
& \theta(p(z))+z p^{\prime}(z) \phi(p(z)) \\
& \quad \prec \theta(q(z))+z q^{\prime}(z) \phi(q(z)) .
\end{aligned}
$$

From Lemma 3, we get

$$
p(z) \prec q(z) .
$$

Thus, we get assertion (69) of Theorem 9.

Theorem 10. Suppose that $0 \leq \rho \leq 1, \eta \in \mathbb{C}, \alpha \in \mathbb{C} \backslash$ $\{0\}, \mathfrak{R}(\alpha)>0$; function $q \in \mathscr{A}^{*}$ is univalent in $\mathbb{U}$ with $q(0)=1$. Let function $f \in \mathscr{H}[0, p]$ satisfy

$$
\begin{aligned}
& {\left[(1-\rho) z \mathscr{W}_{s, b} f(z)+\rho z \mathscr{W}_{s+1, b} f(z)\right] \neq 0 \quad(z \in \mathbb{U}),} \\
& {\left[(1-\rho) z \mathscr{W}_{s, b} f(z)+\rho z \mathscr{W}_{s+1, b} f(z)\right]^{\eta}} \\
& \quad \in \mathscr{H}[1,1] \cap Q .
\end{aligned}
$$

If $\Xi(z)$ defined by (67) is univalent and satisfies

$$
\alpha q(z)+z q^{\prime}(z) \prec \Xi(z),
$$


then

$$
\left[(1-\rho) z \mathscr{W}_{s, b} f(z)+\rho z \mathscr{W}_{s+1, b} f(z)\right]^{\eta} \prec q(z)
$$

and $q$ is the best subordinant in (78). When $\eta=0$, the left hand side expressions of (78) are interpreted as 1 .

Proof. Suppose that function $p(z)$ is defined by (40). From (41), we get

$$
\alpha(p(z))+z p^{\prime}(z) \phi(p(z))=\Xi(z) .
$$

Hypothesis (77) can be rewritten as

$$
q(z)+\left(\frac{1}{\alpha}\right) z q^{\prime}(z) \prec p(z)+\left(\frac{1}{\alpha}\right) z p^{\prime}(z) .
$$

Then, combining Lemma 5 with $\gamma=1 / \alpha$, we have (78). Theorem 10 follows immediately.

Following that, we display some sandwich-type theorems associated with the operator $\mathscr{W}_{s, b} f(z)$.

Theorem 11. Suppose that functions $q_{1}, q_{2} \in \mathscr{A}^{*}$ are univalent mapping of $\mathbb{U}$ into the right half plane and satisfy conditions

$$
\begin{aligned}
& \qquad q_{1}(0)=q_{2}(0)=1, \\
& \qquad \Re\left(1+\frac{z q_{j}^{\prime \prime}(z)}{q_{j}^{\prime}(z)}-\frac{z q_{j}^{\prime}(z)}{q_{j}(z)}\right)>0 \quad(j=1,2 ; z \in \mathbb{U}) . \\
& \text { Let } 0 \leq \rho \leq 1, \alpha, \eta \in \mathbb{C} \text {, and } f \in \mathscr{H}[0, p] \text { satisfy } \\
& \quad\left[(1-\rho) z \mathscr{W}_{s, b} f(z)+\rho z \mathscr{W}_{s+1, b} f(z)\right] \neq 0 \quad(z \in \mathbb{U}), \\
& \quad\left[(1-\rho) z \mathscr{W}_{s, b} f(z)+\rho z \mathscr{W}_{s+1, b} f(z)\right]^{\eta} \\
& \quad \in \mathscr{H}[1,1] \cap Q .
\end{aligned}
$$

If function $\Delta(z)$ is given by (50) and satisfies

$$
q_{1}(z)+\frac{z q_{1}^{\prime}(z)}{q_{1}(z)} \prec \Delta(z) \prec q_{2}(z)+\frac{z q_{2}^{\prime}(z)}{q_{2}(z)},
$$

then

$$
\begin{aligned}
q_{1}(z) & \prec\left[(1-\rho) z \mathscr{W}_{s, b} f(z)+\rho z \mathscr{W}_{s+1, b} f(z)\right]^{\eta} \\
& \prec q_{2}(z),
\end{aligned}
$$

where $q_{1}$ and $q_{2}$ are, respectively, the best subordinant and the best dominant in (84). result.

Combining Theorems 9 and 10, we get the following

Corollary 12. Suppose that $0 \leq \rho \leq 1, \eta \in \mathbb{C}$, and $\alpha \in \mathbb{C} \backslash\{0\}$ with $\mathfrak{R}(\alpha)>0$. Functions $q_{1}$ and $q_{2}$ are univalent convex in $\mathbb{U}$ with $q_{1}(0)=q_{2}(0)=1$. Let $f \in \mathscr{H}[0, p]$ satisfy

$$
\begin{aligned}
& {\left[(1-\rho) z \mathscr{W}_{s, b} f(z)+\rho z \mathscr{W}_{s+1, b} f(z)\right] \neq 0 \quad(z \in \mathbb{U})} \\
& {\left[(1-\rho) z \mathscr{W}_{s, b} f(z)+\rho z \mathscr{W}_{s+1, b} f(z)\right]^{\eta}} \\
& \quad \in \mathscr{H}[1,1] \cap Q .
\end{aligned}
$$

If function $\Xi(z)$ is given by (67) and satisfies

$$
q_{1}(z)+z q_{1}^{\prime}(z) \prec \Xi(z) \prec \alpha q_{2}(z)+z q_{2}^{\prime}(z),
$$

then

$$
\begin{aligned}
q_{1}(z) & \prec\left[(1-\rho) z \mathscr{W}_{s, b} f(z)+\rho z \mathscr{W}_{s+1, b} f(z)\right]^{\eta} \\
& \prec q_{2}(z),
\end{aligned}
$$

where $q_{1}$ and $q_{2}$ are, respectively, the best subordinant and the best dominant in (87).

\section{Conclusions}

In the present paper, making use of the integral operator $\mathscr{W}_{s, b} f(z)$ involving the Hurwitz-Lerch Zeta function, we have derived several third-order differential subordination and differential superordination consequences of meromorphic functions in the punctured unit disk. Furthermore, the sandwich-type theorems are considered. These subordinate relationships have shown the upper and lower bounds of the operator in the punctured unit disk.

\section{Conflicts of Interest}

The authors declare that there are no conflicts of interest regarding the publication of the paper.

\section{Acknowledgments}

The present investigation was supported by the National Natural Science Foundation under Grant no. 11301008, the Foundation for Excellent Youth Teachers of Colleges and Universities of Henan Province under Grant no. 2013GGJS146, and the Foundation of Educational Committee of Henan Province under Grant no. 17A110014.

\section{References}

[1] H. M. Srivastava and J. Choi, Series Associated with the Zeta and Related Functions, Kluwer Academic, London, UK, 2001.

[2] H. M. Srivastava and J. Choi, Zeta and q-Zeta Functions and Associated Series and Integrals, Elsevier, Amsterdam, Netherlands, 2012.

[3] J. Choi and H. M. Srivastava, "Certain families of series associated with the Hurwitz-Lerch zeta function," Applied Mathematics and Computation, vol. 170, no. 1, pp. 399-409, 2005.

[4] M. Garg, K. Jain, and H. M. Srivastava, "Some relationships between the generalized Apostol-Bernoulli polynomials and Hurwitz-Lerch zeta functions," Integral Transforms and Special Functions, vol. 17, no. 11, pp. 803-815, 2006.

[5] S.-D. Lin and H. M. Srivastava, "Some families of the HurwitzLerch zeta functions and associated fractional derivative and other integral representations," Applied Mathematics and Computation, vol. 154, no. 3, pp. 725-733, 2004.

[6] H. M. Srivastava, M. Luo, and R. K. Raina, "New Results Involving a Class of Generalized Hurwitz-Lerch Zeta Functions and Their Applications," Turkish Journal of Analysis and Number Theory, vol. 1, no. 1, pp. 26-35, 2013. 
[7] H. M. Srivastava and A. A. Attiya, "An integral operator associated with the Hurwitz-Lerch zeta function and differential subordination," Integral Transforms and Special Functions, vol. 18, no. 3-4, pp. 207-216, 2007.

[8] J.-L. Liu, "Sufficient conditions for strongly star-like functions involving the generalized Srivastava-Attiya operator," Integral Transforms and Special Functions, vol. 22, no. 2, pp. 79-90, 2011.

[9] Z.-G. Wang, Z.-H. Liu, and Y. Sun, "Some properties of the generalized Srivastava-Attiya operator," Integral Transforms and Special Functions, vol. 23, no. 3, pp. 223-236, 2012.

[10] Y. Sun, W.-P. Kuang, and Z.-G. Wang, "Properties for uniformly starlike and related functions under the Srivastava-Attiya operator," Applied Mathematics and Computation, vol. 218, no. 7, pp. 3615-3623, 2011.

[11] S.-M. Yuan and Z.-M. Liu, "Some properties of two subclasses of k-fold symmetric functions associated with Srivastava-Attiya operator," Applied Mathematics and Computation, vol. 218, no. 3, pp. 1136-1141, 2011.

[12] Z.-G. Wang and L. Shi, "Some subclasses of meromorphic functions involving the Hurwitz-Lerch zeta function," Hacettepe Journal of Mathematics and Statistics, vol. 45, no. 5, pp. 14491460, 2016.

[13] K. R. Alhindi and M. Darus, "A new class of meromorphic functions involving the polylogarithm function," Journal of Complex Analysis, vol. 2014, Article ID 864805, 5 pages, 2014.

[14] A. Y. Lashin, "On certain subclasses of meromorphic functions associated with certain integral operators," Computers \& Mathematics with Applications, vol. 59, no. 1, pp. 524-531, 2010.

[15] J. A. Antonino and S. S. Miller, "Third-order differential inequalities and subordinations in the complex plane," Complex Variables and Elliptic Equations. An International Journal, vol. 56, no. 5, pp. 439-454, 2011.

[16] S. S. Miller and P. T. Mocanu, "Subordinants of differential superordinations," Complex Variables, Theory and Application, vol. 48, no. 10, pp. 815-826, 2003.

[17] H. Tang, H. M. Srivastava, S.-H. Li, and L.-N. Ma, "Thirdorder differential subordination and superordination results for meromorphically multivalent functions associated with the Liu-Srivastava operator," Abstract and Applied Analysis, vol. 2014, Article ID 792175, 2014.

[18] S. S. Miller and P. T. Mocanu, Differential Subordinations: Theory and Applications, vol. 225 of Monographs and Textbooks in Pure and Applied Mathematics, Marcel Dekker, New York, NY, USA, 2000.

[19] S. S. Miller and P. T. Mocanu, "On some classes of first-order differential subordinations," Michigan Mathematical Journal, vol. 32, no. 2, pp. 185-195, 1985.

[20] S. S. Miller and P. T. Mocanu, "Briot-Bouquet differential superordinations and sandwich theorems," Journal of Mathematical Analysis and Applications, vol. 329, no. 1, pp. 327-335, 2007. 


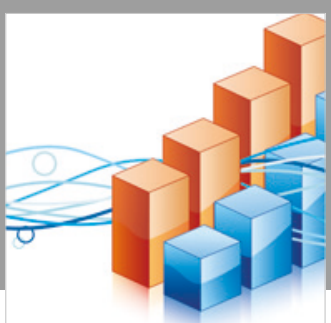

Advances in

Operations Research

\section{-n-m}
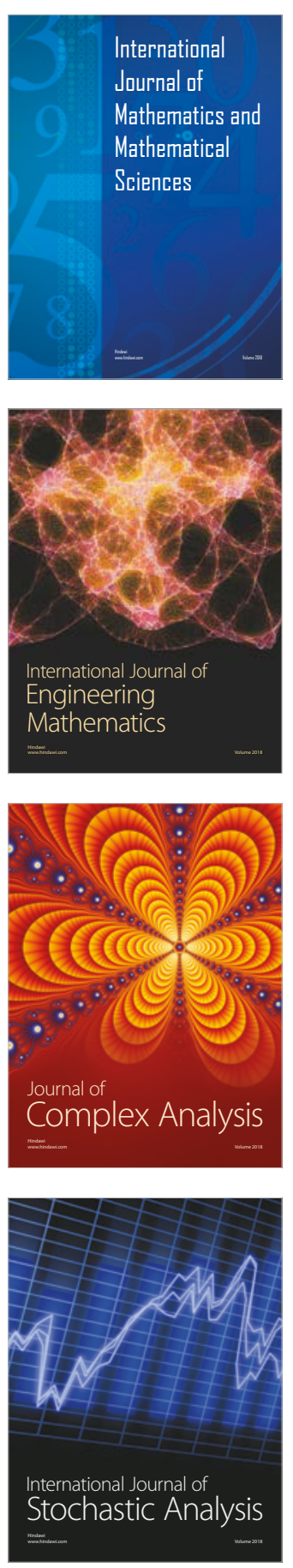
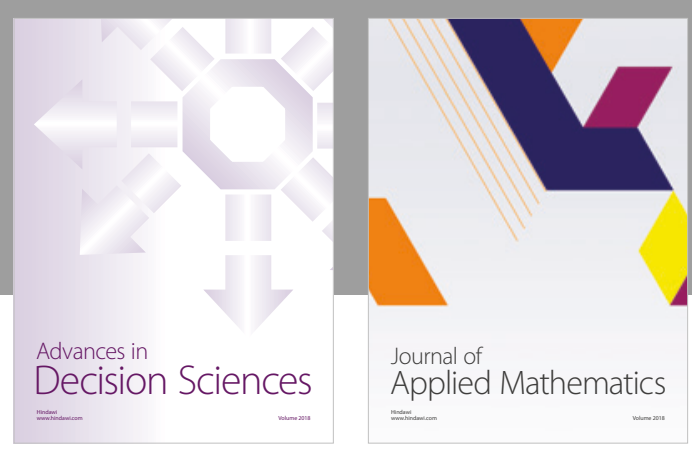

Journal of

Applied Mathematics
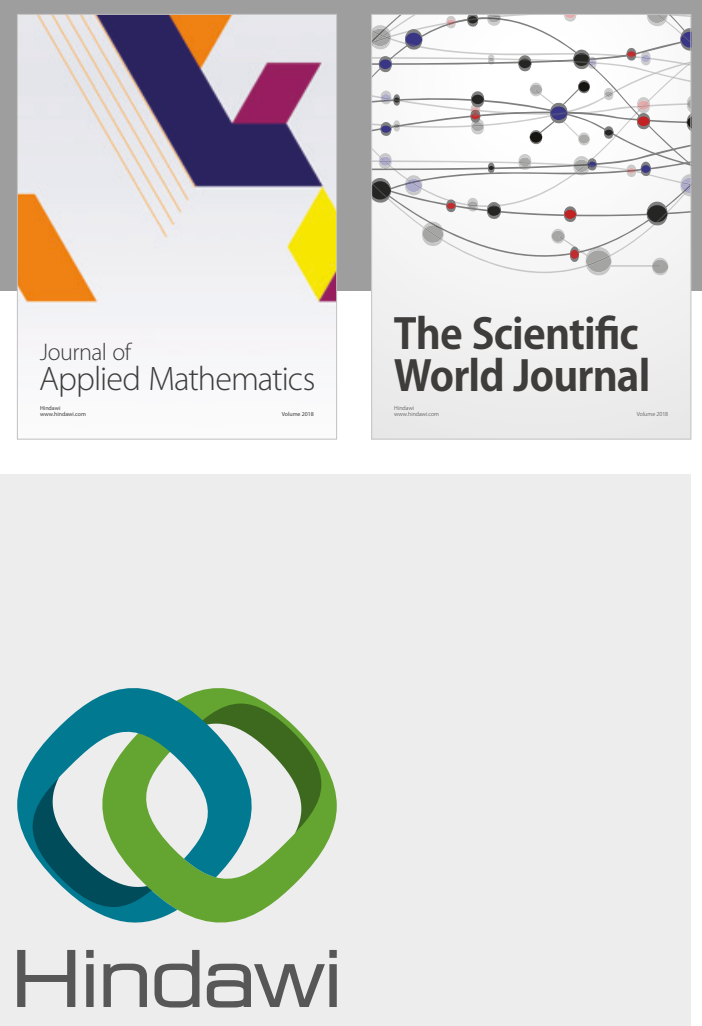

Submit your manuscripts at

www.hindawi.com

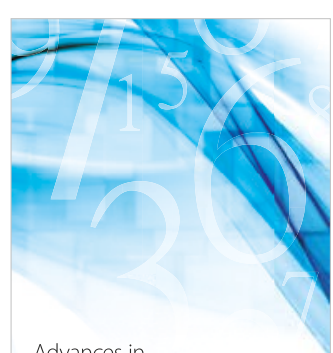

Advances in
Numerical Analysis
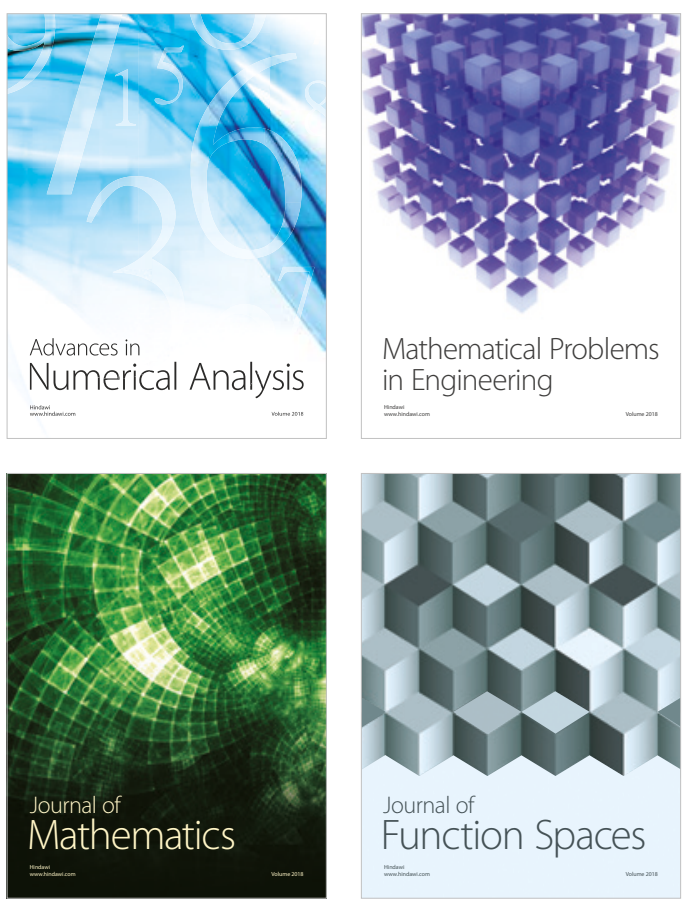

Mathematical Problems in Engineering

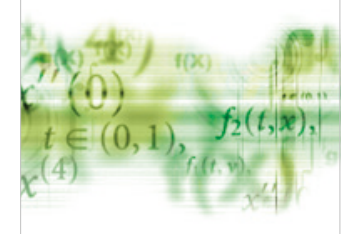

International Journal of

Differential Equations

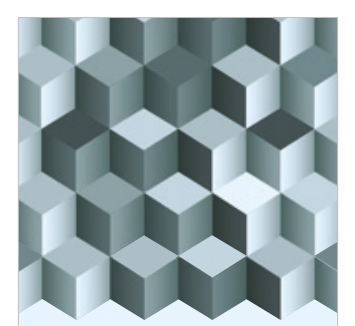

Journal of

Function Spaces

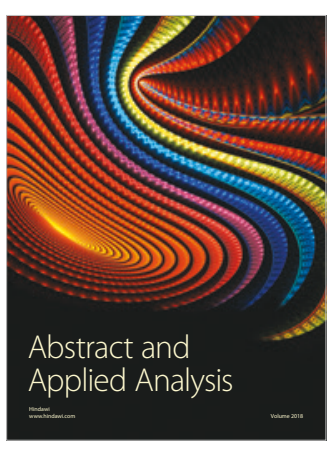

The Scientific

World Journal

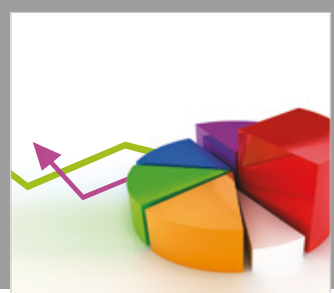

Journal of

Probability and Statistics
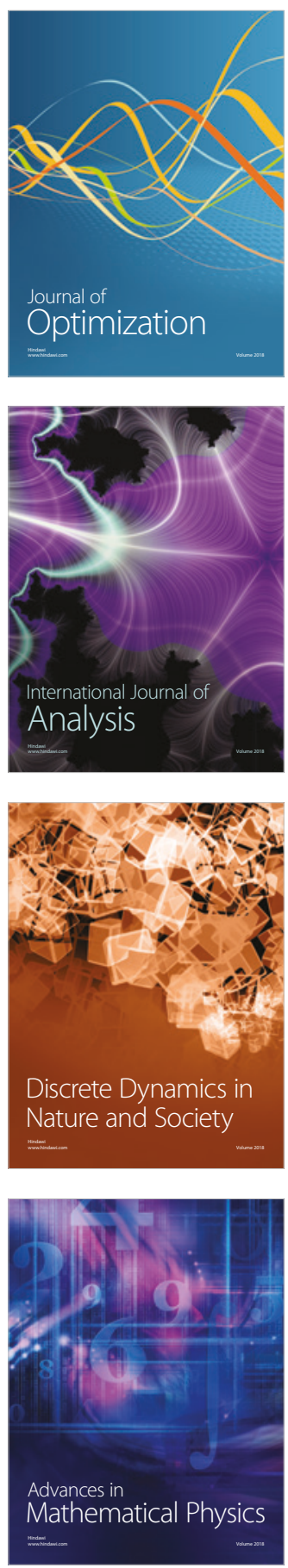